\title{
El uso de las redes sociales como fuente de información en situaciones de riesgo ciudadano
}

Recibido: 13 de octubre de 2014

Aceptado: 06 de abril de 2015

\author{
Mercedes Cancelo Sanmartín \\ cancelo@uma.es \\ Universidad de Málaga (España) \\ Navid González Márquez \\ alejandra_glz_marq@hotmail.com
}

Universidad Da Vinci (México)

Resumen: Las instituciones abocadas a la seguridad ciudadana tienen entre sus obligaciones mantener informada a la ciudadanía sobre los diversos acontecimientos referidos a la seguridad del entorno. Cuando este vínculo entre instituciones y sociedad se fragmenta, los ciudadanos buscan fuentes de información alternativas, como son las redes sociales en línea, que en la actualidad parecen ser confiables debido a la legitimación de sus públicos, convirtiéndolos en líderes de información y opinión. No obstante, en la presente investigación, se debe considerar que la percepción de los individuos (sobre la base de la información que fluye a través de las redes sociales tradicionales como en línea) puede crear fenómenos de desinformación y victimización.

Palabras clave: Redes sociales, instituciones, percepción, victimización, crisis.

Abstract: Institutions committed to public safety are among its obligations to keep the public informed about various events related to the safety hazards in the environment. When the link between institutions and society is fragmented, citizens seek alternative sources of information as they are online social networks that currently seems to be reliable because the legitimacy of their public, making information and opinion leaders. However, this investigation should be considered that the perception of individuals based on the information that flows through traditional social networks and online turn can create phenomena of misinformation and victimization.

Key words: Social Networks, Institutions, Perception, Victimization, Crisis. 


\section{Introducción}

En el Estado de Tamaulipas (México) la falta de acceso a la información oficial, con respecto a los sucesos derivados de hechos delictivos (desde el año 2010), originó que una parte de los ciudadanos recurrieran a las redes sociales (tanto tradicionales como en línea) para satisfacer sus necesidades de conocimiento del entorno. Sin embargo, a través de la red se dan situaciones de sobrecarga de información con versiones encontradas, hechos inexistentes y otros verídicos, captados por ciudadanos y difundidos a través de las redes por Internet o por conversaciones del tema. Esto ha provocado un fenómeno de desinformación y confusión dentro de la sociedad en muchos de los casos.

Los individuos y grupos tienden a buscar líderes en situaciones de crisis y sus necesidades específicas determinarán quién o quiénes serán seleccionados como tales. Si las necesidades de información no son satisfechas por las fuentes formales (en este caso las instituciones públicas), las informales tendrán un peso fundamental en la retransmisión de información informal: así, resultaría importante dar al receptor la información, ya no lo que desconoce (puesto que aunque la fuente no sea confiable, existe un precedente, como noción previa de información) sino la información que se relacione con los hechos más cercanos a la realidad que se suscitan en esos momentos.

Este esquema de comunicación propicia un círculo vicioso que genera que las instituciones sean apreciadas como inconsistentes, en especial las vinculadas con la prevención de los delitos. El entender la naturaleza de esta percepción, así como los roles de los individuos que generan los flujos de información informal, son las principales motivaciones de este estudio, así como el entendimiento de estos flujos en la creación de confianza y legitimización en las instituciones.

\section{Objetivos}

Los objetivos marcados en esta investigación son los siguientes:

- Analizar la percepción de los ciudadanos sobre la difusión de los hechos delictivos por parte de las instituciones públicas.

- Analizar la información que fluye en las redes sociales en línea sobre los hechos delictivos y su efecto en la percepción de la población objetivo.

\section{Metodología}

En cuanto a la muestra para el estudio, se seleccionó a jóvenes de 18 a 29 años, quienes representan el $26.1 \%$ de la población de Tamaulipas, aunados con la delimitación preferente de alumnos de nivel superior de las diversas universidades del Estado, debido a su mayor alcance, inmersión e influencia dentro de las redes sociales tradicionales y en línea. 
- La selección de esta muestra se llevó a cabo debido a sus características sobre el uso de redes sociales en línea, así como las redes sociales tradicionales, debido a que:

- Que por su rango de edad, se encuentran inmersos en diversas redes sociales tradicionales, no solo son universitarios: algunos tienen un trabajo, forman lazos fuertes y débiles en los procesos educativos extracurriculares, deportivos, religiosos, entre otras.

- A su vez, por estas redes tradicionales tienen la necesidad de estar en contacto, por lo cual son proclives a la utilización y aceptación de las nuevas tecnologías (redes sociales en línea) como cambio a los paradigmas de los medios tradicionales.

- Se encuentran concentrados en el mismo espacio virtual: las redes sociales.

- La primera etapa consistió en seleccionar las unidades del muestreo probabilístico por racimos, con acceso propio o comunitario a las redes sociales en línea y que estén inmersos en diversas redes sociales tradicionales. El marco de muestra se basó en la lista de escuelas existentes, mediante bases de datos obtenida a través de la Secretaria de Educación Pública de México (SEP).

La selección de la muestra y aplicación del instrumento presentó dos particularidades:

- Se aplicó el instrumento dividiendo el estado en tres zonas: norte, centro y sur.

- Por características propias de la temática, se realizó la convocatoria a través de la red social en línea Facebook. Se creó una encuesta, a través del servidor e-encuesta.

El tipo de estudio es no experimental descriptivo y el alcance del estudio es exploratorio, debido a que la investigación dentro de la literatura existente ha demostrado que el estudio de la transmisión de información y el comportamiento de las personas (en un clima que perciben como inseguro, aunque se ha estudiado anteriormente) no considera las variables de las nuevas tecnologías (como elemento de comunicación) ni su injerencia en la apreciación del ambiente del individuo, a la par de la variable de victimización, con relación a la comunicación o falta de esta de la instituciones públicas y posibilidad de disminuir su legitimización con relación a la percepción ciudadana.

\section{Hipótesis}

Como hipótesis de investigación se plantearon dos con el fin de conformar una investigación lo suficientemente completa que arrojase resultados probados y confiables:

- En situaciones de inseguridad pública, los ciudadanos buscan a las instituciones oficiales como fuentes primarias de información; a falta de información oficial, los individuos recurren a fuentes informales.

- La falta de información oficial en situaciones de hechos delictivos conlleva a la no legitimización de las instituciones gubernamentales en Tamaulipas (México). 


\section{Opinión pública}

González (1997: 52) expone que "el mensaje enviado será efectivo en la medida que sea entendido por el receptor". Efectivamente, el significado está ligado a una variedad de estímulos, a los que se responde de acuerdo a la experiencia y la información disponible. La información se transmitirá según la percepción de los diversos actores que participan en el proceso de la comunicación. Es por ello, que esta acción comunicativa generará opinión pública. Para Sartori el concepto de opinión pública deriva de "un público, o multiplicidad de públicos, cuyos difusos estados mentales de opinión se interrelacionan con corrientes de información referentes al estado de la res pública" (ápud Botero y Correa, 2006: 81).

Lippmann (2010) afirma que la opinión pública es una realidad de segunda mano, que empieza por una ventana al mundo a través de los medios de comunicación y la formación de ésta se basa en la visión o perspectiva del hecho o acción que culmina con la implementación de una determinada decisión en donde intervienen elementos culturales y de experiencia individual. Asimismo, destaca que democracia y opinión pública van de la mano. El contraste empieza desde el concepto opinión que se refiere a algo personal, íntimo y público hace referencia a la apertura hacia la colectividad. A la vez, el contraste está en la distorsión a la atribución del significado de lo público.

La construcción de la opinión pública, con base a su conceptualización, es un proceso que empieza con la formación de una idea, la cual es moldeada y cambia continuamente debido a la información que surge con el tiempo. Botero y Correa (2006) indican la intervención de tres procesos en la formación de opinión pública: a) La forma de las creencias, b) actitudes y c) la percepción de algún tipo de apoyo social.

En la figura 1 se puede observar cómo el proceso comunicativo se desarrolla para la conformación de la opinión pública, de acuerdo con lo que Botero y Correa (2006: 159) señalan sobre la perspectiva de los públicos.

Figura 1: La formación de la opinión pública.

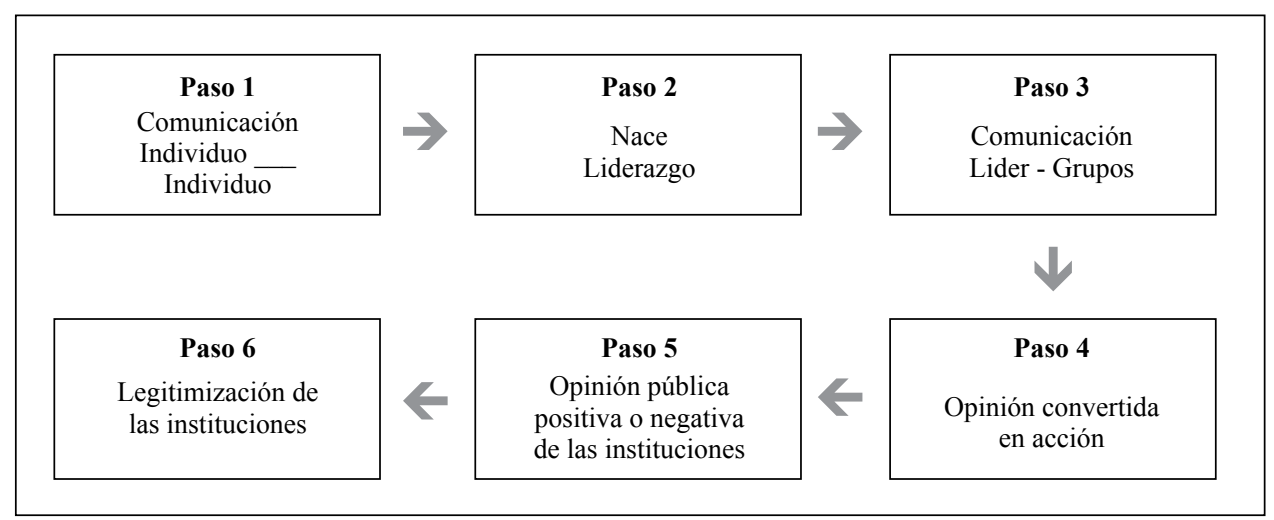

Fuente: Elaboración propia, basada en Botero y Correa (2006). 


\section{Comunicación institucional}

La comunicación institucional se define como el tipo de comunicación "realizada de modo organizado por una institución o sus representantes, y dirigida a las personas y grupos del entorno social en el que desarrolla su actividad" (La Porte, 2005). Este tipo de comunicación no existe desde la aparición de las instituciones sino que inicialmente se generó un proceso puramente informativo, es en las últimas dos décadas cuando podemos hablar de la generación de la comunicación desde las instituciones.

La Porte (2012: 281-282) describe el flujo de la comunicación institucional en siete etapas, que giran en torno al principio básico de cada institución que a continuación se describen:

- "Identidad: se refiere a la institución. Considera las características de la institución que la diferencia de otras.

- Misión: es el fin, la razón de ser y lo que hace, sus objetivos para la satisfacción de las necesidades de los ciudadanos.

- Cultura: es la esencia de las organizaciones, sus ideas, valores, normas, políticas, entre otros; que distinguen las relaciones entre trabajadores de la institución y ciudadanos.

- Imagen: se refiere a cómo la ven. Es la percepción de los ciudadanos hacia la institución en el rubro de la prestación de su servicio. Ésta se compone de dos elementos: comportamiento y actitud de las personas y coherencia entre lo prometido y lo realizado.

- Reputación: a través del tiempo las Instituciones pueden tener una evaluación positiva o negativa de sus públicos e incluso su confianza y respeto. Se refiere a como los públicos la valoran.

- Autoridad: es la cúspide de la legitimización de la Institución ante la ciudadanía, debido a que reconocen su utilidad, y admiran la labor que realiza hacia la sociedad".

La comunicación institucional tiene sus raíces en los modelos de relaciones públicas y de comunicación organizacional. No obstante, va más allá de estas funciones debido a que estimula el diálogo y las relaciones entre los ciudadanos e instituciones. La identificación de los públicos y su aceptación (además de que la percepción) se vuelve un proceso fundamental. Hablar de aceptación significa hablar de legitimización. Weber (1984: 701) se refiere a la base de la legitimidad como "la vigencia del poder, es decir, la pretensión de que el gobernante sea obedecido por sus funcionarios y todos ellos por los gobernados". Utiliza el concepto desde la vía de la dominación social, el poder y las relaciones sociales de los actores que interactúan en una sociedad.

Este no es un proceso voluntario, sino más bien, se refiere al conjunto de ideas de un colectivo, y a la relación entre estas ideas con los actores gubernamentales, además de 
la aceptación o trasformación a nuevas temáticas que pasan a formar parte de la opinión pública, las cuáles serán fiscalizadas a su vez por el liderazgo de determinados actores, que participarán activamente en el proceso.

En este punto es cuando se habla de legitimidad. Se debe agregar también implícitamente los conceptos de "reconocimiento" y "utilidad". En el caso de la comunicación institucional su función es construir una buena realidad de las instituciones a través la comunicación, mostrando a la sociedad sus acciones, resultados y fortalezas para conquistar la confianza de sus públicos y por lo tanto su aceptación ante la opinión pública. Tal y como indica Gadea (2014: 69) la comunicación institucional asertiva "es fundamento primordial para el buen funcionamiento y pervivencia de la institución en el marco de la sociedad en la que está inmersa".

\section{Crisis de comunicación en instituciones públicas y nuevas tecnologías}

Para Losada (2010: 24-25), crisis se denomina a "una situación más o menos excepcional e inesperada y potencialmente dañina". Los directores de comunicación en las instituciones deben coordinar los mensajes que se envían al exterior, debido a que una multiplicidad de mensajes dificultará la comunicación con los públicos a los que desea llegar. La institución deberá ser una fuente de información, no de confusión.

En diversos textos se han señalado las necesidades de información de las instituciones, de los públicos y de los datos de su entorno que son sumamente importantes. Pero ¿qué hacen los públicos cuando la institución e incluso los medios no les proporcionan la información necesaria? Como se ha comentado anteriormente, ante la falta de comunicados y fuentes formales, surgirán las fuentes informales en línea, las que en la presente investigación se les clasificará en dos categorías:

- Fuentes informales de información: son fuentes que trasmiten datos corroborados y verídicos de los sucesos que les interesan a los diversos públicos que conforman la sociedad, que son comprobables e incluso exhiben fotografías de los hechos y que a través de la aceptación de los usuarios se han ganado la legitimización como fuentes confiables de información.

- Fuentes informales de desinformación: son fuentes de trasmisión de datos que no están confirmados ni legitimizados. Su retransmisión suele ser el paso siguiente, por lo cual se generará más desinformación. Se entiende como desinformación: "La situación en la que, por incumplimiento de las normas lingüísticas y/o profesionales, el destinatario de la información recibe un producto informativo incorrecto a consecuencia del silencio, la intencionalidad o el error de la fuente" (Rivas, 1995: 78).

Una vez que surgen las crisis de comunicación, por cualquier tipo de las fuentes mencionadas anteriormente, es necesario realizar un estudio, reconociendo en primer lugar la existencia del problema viendo los escenarios existentes desde donde surge. Este es 
un apartado importante en el caso de las nuevas tecnologías. Sin embargo, durante los inicios de un proceso de crisis (aunado a la rápida reproducción de la información a través de herramientas como redes sociales, teléfonos con acceso a Internet, correo electrónico entre otros) ignorarla lleva a los ciudadanos a preguntarse: ¿Si no es verdad, entonces, por qué no se desmiente? En tópicos como hechos que ponen en peligro la integridad de los individuos (moral, física, económica, etc.) es todavía mayor la necesidad de darle al público información oficial veraz y oportuna. Aquí es donde es posible utilizar las nuevas tecnologías como una ventaja ante la desinformación.

Losada (2010) define dos clases de sucesos en la crisis: a) sucesos previsibles, que se detectan riesgos potenciales y rutas específicas de manejo de esos sucesos manual; y $b$ ) sucesos imprevisibles, que acontecen sin previo aviso y que bajo su naturaleza aparecen sucesos específicos. La diferencia entre estos radica en las etapas de crisis y poscrisis debido a que el resultado de estas en los sucesos imprevisibles son acciones tardías o por lo general variables que no estaban contempladas al momento de la crisis.

Cuando se habla de poscrisis nos referimos a que la crisis no es el final de la historia, sino que hay un período en el que es necesario volver a ser aceptado (restauración de la reputación) y en donde la información y su transmisión juegan un papel importante. Asimismo, destaca que en esta fase se debe de prestar atención a los públicos perjudicados, estar comunicados con los medios y comunicar a su vez a estos si hay acciones correctivas en proceso, analizar el plan de crisis y su función, crear una retroalimentación de la experiencia y crear planeación a corto y largo plazo para el análisis y reparación de daños por las crisis.

A continuación se señalan algunos modelos de crisis. A pesar de que existen más modelos, se considera que estos pueden ser aplicados en instituciones públicas:

Figura 2: Modelos de crisis aplicables a instituciones públicas.

\begin{tabular}{|c|c|c|}
\hline MODELO & AUTOR & FASES \\
\hline Siete capas & Hurd & $\begin{array}{l}\text { - Conectividad reuniones iniciales del equipo de crisis. } \\
\text { - Corrección de datos obtener información sobre el daño de las crisis } \\
\text { - Filtrado convertir los datos disponibles en información útil para la } \\
\text { gestión de crisis. } \\
\text { - Elección de los medios correctos para que la información llegue de } \\
\text { forma correcta al receptor. } \\
\text { - Memoria organizacional tomar los datos más útiles de crisis } \\
\text { anteriores. } \\
\text { - Valores establecen prioridades en las crisis. } \\
\text { Proceso de grupo tomar todos los datos anteriormente mencionados } \\
\text { para toma de decisiones. }\end{array}$ \\
\hline
\end{tabular}




\begin{tabular}{|l|l|l|}
\hline $\begin{array}{l}\text { Relaciones } \\
\text { públicas de crisis }\end{array}$ & Marra & $\begin{array}{l}\text { - Planificación de la comunicación de crisis. } \\
\text { - Análisis de las relaciones previas con los públicos. }\end{array}$ \\
\hline Gestión proactiva & $\begin{array}{l}\text { González } \\
\text { Herrero }\end{array}$ & $\begin{array}{l}\text { Ciclo de vida de las crisis. Parecido al ciclo de vida biológico, las crisis } \\
\text { pasan por la etapa de nacimiento, crecimiento, madurez y muerte. } \\
\text { Identificación de asuntos potencialmente conflictivos, que identificados } \\
\text { a tiempo no derivan en crisis. No llamar la atención de los medios. }\end{array}$ \\
\hline
\end{tabular}

Fuente: Losada (2010) y González Herrero (2008).

\section{Redes sociales}

Dentro de este estudio, se definen dos conceptos de redes sociales: $a$ ) redes sociales en línea y b) redes sociales tradicionales. La utilización de las últimas nace de la mano de la sociología, psicología y las matemáticas, con el análisis de la comunicación entre grupos. Se define como grupo al "conjunto de personas que interactúan entre sí, de modo que cada miembro recibe la influencia de cada una de las otras personas y a su vez, ejerce influencia en ellas" (Fernández Collado, 2001: 15). Es decir debe existir una relación entre los sujetos y por lo menos un vínculo que los una entre ellos, que sea cuantificable. Cuando hablamos de sujetos, no nos limitamos a personas, sino también, a organizaciones, países e instituciones, entre otros.

Este acercamiento entre grupos es el objetivo que instituye a las redes sociales. Estas redes surgen de necesidades afectivas, de interacción y socialización de individuo, por ello se componen de personas con patrones de conducta similares, afirmando diversos teóricos como Neumann (1995), que los grupos se conformarán de individuos con intereses similares entre sí. La principal desventaja de las redes sociales tradicionales es que las relaciones entre individuos se basan en el trato indirecto, es decir, siempre hay intermediarios, por lo cual el proceso de comunicación es más dilatado en el tiempo y puede ser menos confiable debido a que se basa en reglas de los grupos.

Las redes sociales en línea, basan su desarrollo en el avance tecnológico de lo que suele llamarse web 2.0. En contraposición de la web 1.0, los usuarios pueden interactuar con su entorno en la red, a través de comunidades. Tienen su antecedente en los sitios online, que se comunicaban a través de sistemas de boletines en tableros (BBS). Los usuarios se comunicaban entre muros y participaban en discusiones.

Elison (2007) define a las redes sociales en línea como servicios basados en la web que permiten a los individuos:

- “Construir un perfil público o semipúblico dentro de un sistema delimitado.

- Articular una lista de otros usuarios con quienes comparten una conexión. 
- Ver y recorrer su lista de conexiones y las hechas por otros dentro del sistema. La naturaleza y la nomenclatura de estas conexiones pueden variar de un sitio a otro".

Amidon (2011) sostiene que las redes sociales en línea han pasado a formar parte de nuestra rutina, y que incluso las personas han creado su propio espacio en la red. Esto debido a que la infraestructura tecnológica ha avanzado a pasos agigantados en los últimos 3 años, permitiendo no solo la conexión a través de la computadora, sino a través de otros dispositivos. A diferencia de las redes sociales físicas o tradicionales, las redes sociales en la Internet presentan un acceso directo entre usuarios. Según Orihuela (2008) las actividades básicas realizadas en las redes sociales y sus usos por los usuarios son:

- Vías de comunicación directa: con cualquier usuario, a cualquier hora, sin necesidad de la intervención de un tercero.

- Cooperación: los ejemplos más significativos de esta clase de transmisión de información son los terremotos en Chile y en Haití, pues a través de las redes sociales, hubo comunicación entre ellos y el mundo, pues a la magnitud del temblor, medios como el teléfono e incluso las televisoras locales dejaron de funcionar. Asimismo, también se utilizan como medios para divulgar información acerca de eventos colectivos, como el ejemplo de la manifestación masiva en apoyo a los padres de los niños fallecidos en la guardería $\mathrm{ABC}$.

- Comunidad: en cuanto a su alcance a usuarios y miembros, veamos la siguiente tabla en relación a los usos y costumbres de los internautas mexicanos (figura 3), de los cuales se tomaron los datos relevantes en cuanto al uso de redes sociales en línea:

Figura 3: Cifras de las principales redes sociales.

\begin{tabular}{|l|c|c|c|}
\hline RED & \% DE USUARIOS 2011 & $\begin{array}{l}\text { \% DE USUARIOS } \\
\mathbf{2 0 1 2}\end{array}$ & \% DE AUMENTO \\
\hline Facebook & $39 \%$ & $90 \%$ & $51 \%$ \\
\hline YouTube & $28 \%$ & $60 \%$ & $32 \%$ \\
\hline Twitter & $20 \%$ & $55 \%$ & $35 \%$ \\
\hline $\begin{array}{l}\text { \% de Mexicanos que utilizan } \\
\text { las redes sociales }\end{array}$ & $61 \%$ & $92 \%$ & $31 \%$ \\
\hline $\begin{array}{l}\text { Tiempo de conexión a } \\
\text { Internet }\end{array}$ & 3 horas y 42 minutos & 4 horas y 9 minutos & 27 minutos \\
\hline $\begin{array}{l}\text { Lugar de principal actividad } \\
\text { online (\%) }\end{array}$ & Cuarto $61 \%$ & Segundo $77 \%$ & $16 \%$ \\
\hline $\begin{array}{l}\text { Principal elemento de } \\
\text { conectividad }\end{array}$ & Teléfono celular 26\% & Smartphones 58\% & $32 \%$ \\
\hline
\end{tabular}

Fuente: Asociación Mexicana de Internet (2011). 
Como se observa, el uso de las principales redes sociales en línea se ha triplicado y no solo se utilizan para cuestiones de entretenimiento, sino también en el uso de las mismas con propósitos profesionales, de marketing o de difusión masiva de una idea. En cuanto a su naturaleza, podemos dividir las funciones de las redes sociales en línea en:

- Fuentes de entretenimiento.

- Fuentes de envió/recepción de mensajes.

- Canalizadores de información.

- Canales de búsqueda de necesidades concretas (búsqueda de empleo).

- Fuentes de información en situación de crisis (información de hechos delictivos).

\section{Percepción y victimización}

Para que exista una interacción social es necesario que las personas se perciban mutuamente. La percepción ayuda a entender los sucesos a nuestro alrededor y darles una explicación. Se trata de predecir lo que otros harán o dirán, y racionalizar sus motivos. Se observa la información que tenemos y la manera en que conseguimos dicha información.

Al hablar de percepción nos referimos inmediatamente a comportamiento; sin embargo, factores genéticos, la evolución social y el entorno son elementos que orientan y animan nuestra percepción. Un medio cultural puede afectar la realidad y la estimulación al respecto. Tajfel (1969) ha determinado tres criterios sobre los factores sociales y culturales de la percepción:

- "Familiaridad: frecuencia de contacto con un estímulo de una cultura que hace que aparezcan hábitos perceptuales. Nuestras experiencias pasadas facilitan la percepción de los estímulos con los que tuvimos contacto anteriormente. La familiaridad crea una disposición a una respuesta inmediata. Los estímulos conocidos son más fácilmente comunicables y se pueden aprovechar determinadas disposiciones a responder para una mayor eficacia de determinada comunicación persuasiva

- Valor funcional: la importancia de las distintas propiedades del ambiente, que hacen desarrollar dispositivos selectivos, predisposición o rechazo frente a la estimulación.

- Sistemas de comunicación: el medio desarrolla información que se categoriza mediante códigos".

Es de importancia analizar no solo la percepción individual sino también la percepción de grupos, ya que intervienen tanto en la percepción individual como en la personalidad individual de sus integrantes. Aquí es donde entramos en el apartado de victimización. La tipología de la víctima conlleva a la clasificación de la victimización. Sin embargo, los límites entre los diferentes tipos de victimización son difusos. 
Ferreiro (2005: 109) señala que existen en la actualidad "determinados factores que están provocando un aumento desproporcionado del miedo al delito, en relación con las probabilidades reales de victimización". El mencionado autor indica que aunque no hay relación entre inseguridad real y ficticia, el hecho de percibir riesgos distorsiona la objetividad del individuo y cambia sus patrones culturales. Pero cuestiona los papeles de los medios de comunicación como desencadenadores de la victimización subjetiva, por su confiabilidad y credibilidad, creando sesgos de información.

El punto contrario es lo que se propone en este trabajo de investigación y es que la falta de información por fuentes formales (ya sea medios escritos, masivos e incluso canales formales de comunicación como instituciones gubernamentales) genera miedo a ser víctima de un hecho delictivo. Este miedo es el sentimiento de ansiedad causado por la posibilidad de ser víctima de un delito, identificando como características principales: el miedo en sí mismo y la sensación de victimización que se concibe.

Este trabajo propone que la victimización no solo va de la mano de la falta de información formal sino también del medio que la sustituye con las nuevas tecnologías, específicamente las redes sociales. Se propone, para este fin, el "modelo de victimización en SDR y las TIC", que presenta los siguientes tipos de victimización:

- Victimización inducida: se da ante la sobrecarga de información o desinformación en las redes sociales tradicionales o en línea (RSEL/T) sobre situaciones de riesgo (SDR), con lo cual el individuo busca datos en los medios formales de información (MMTIG). Como medios masivos tradicionales e instituciones gubernamentales, le proporcionan distractores mediáticos y no cubren sus necesidades.

- Victimización por percepción de SDR: sobrecarga de información que propicia el sentimiento de inseguridad y angustia dentro del entorno del individuo, de solo saber que las condiciones de desarrollo y oportunidades se tornan inexistentes. Esta situación lleva a la aplicación de medidas preventivas que salen de las rutinas tradicionales o incluso que orillan a evitar situaciones convencionales que podrían llevar a una situación de Riesgo (SDR).

- Victimización por falta de filtros de información: cuando el individuo no conoce a la víctima, pero a través de RSEL o redes sociales tradicionales conoce los sucesos que lo llevaron a ser víctima, lo que pasaría en situaciones convencionales sería que podría desechar o tomar la información con mayor objetividad. Asimismo en situaciones de riesgo (aunadas a las lagunas de información oficial y a las necesidades de conocer sobre lo que acontece) el individuo tomará premisas que, por lo general, no haría y le generaría conflictos miedo, desorientación, etc., sin saber si esta información es verdadera o está distorsionada. 


\section{Resultados de la investigación}

En cuanto al cuestionamiento sobre la oferta de información oportuna sobre hechos delictivos por parte de las redes sociales el $86 \%$ de los encuestados estuvieron de acuerdo con esta afirmación; en contraste en la consulta de la oferta de información oportuna sobre hechos delictivos por parte de los medios de comunicación como radio, televisión y prensa, solo el $41 \%$ mostró satisfacción con la trasmisión de datos por estos medios.

Figura 4: Frecuencia de utilización de medios tradicionales y no tradicionales para recibir información de hechos delictivos.

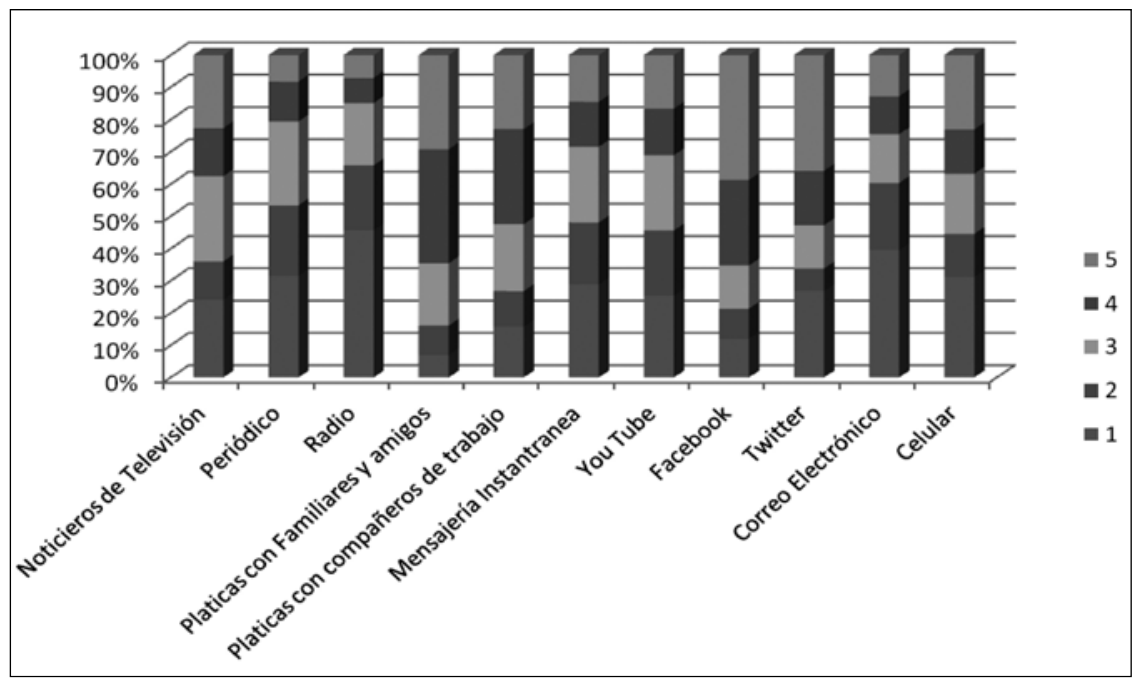

Fuente: Elaboración propia.

Se cuestionó a los entrevistados sobre su percepción de las redes de comunicación como agentes de desinformación, el $10 \%$ está muy de acuerdo con este postulado, el $39 \%$ está de acuerdo, el 32\% está en desacuerdo, el 15\% totalmente en desacuerdo y el $4 \%$ contesto que no está de acuerdo ni en desacuerdo. En cuanto a la afirmación "de las redes sociales en línea que proporcionan información sobre hechos delictivos de manera oportuna", el $21 \%$ de los encuestados está totalmente de acuerdo, seguido del $45 \%$ que está de acuerdo, el 19\% está en desacuerdo, el $9 \%$ totalmente en desacuerdo y el $6 \%$ no está de acuerdo ni en desacuerdo.

\subsection{Redes sociales}

Los medios más utilizados para recibir información de hechos delictivos son las pláticas con familiares y amigos (13\%), Facebook (16\%), pláticas con compañeros de trabajo $(10 \%)$ y Twitter (15\%). Los menos utilizados son el correo electrónico (14\%) y la radio (16\%), seguidos por el periódico y el celular (11\%). 
Figura 5: Nivel de utilización de medios tradicionales y no tradicionales para transmisión de información de redes sociales

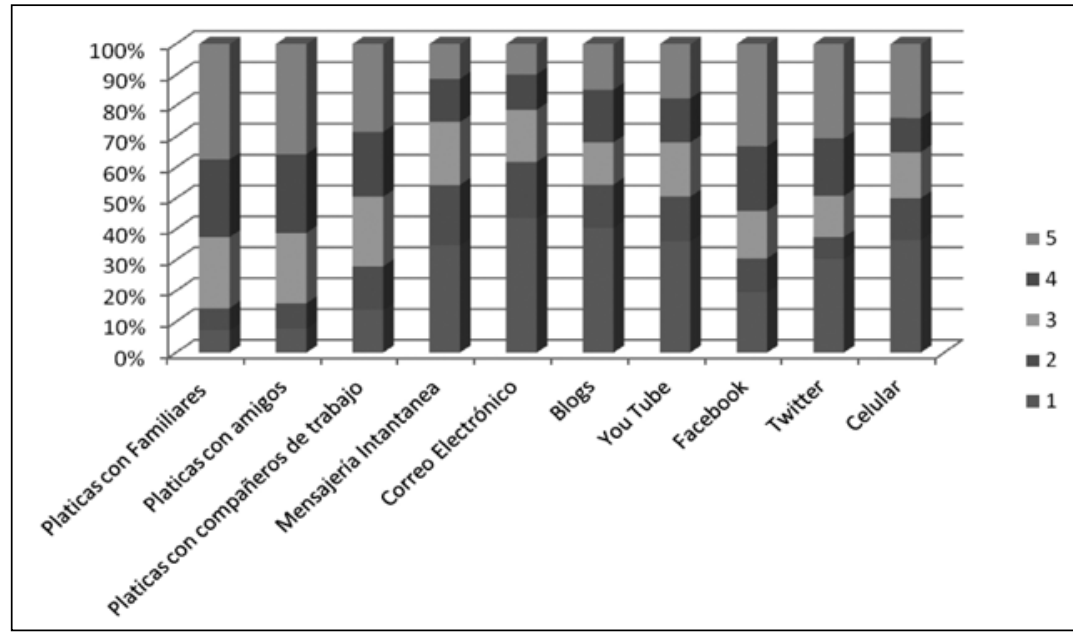

Fuente: Elaboración propia.

Los medios por los cuales se transmite la información son las pláticas con familiares y las pláticas con amigos (ambas con 29\%) y Twitter (25\%); los que se usan menos son el correo electrónico (30\%), la mensajería instantánea (29\%) y los blogs $(26 \%)$.

\subsection{Victimización}

Se presentó una matriz con las siguientes afirmaciones:

- Considero que la situación de inseguridad que se vive actualmente ha cambiado el ritmo de vida del Estado.

El $95 \%$ de la población encuestada, considera cierta esta aseveración.

- La información que recibo a través de las redes sociales me hace pensar que soy propenso a ser víctima de un delito.

El 33\% de los encuestados eligieron la opción "Muy de acuerdo".

El $40 \%$ eligieron la opción "De acuerdo".

El 21\% están en desacuerdo con la afirmación.

El 3\% está totalmente en desacuerdo.

El 3\% restante no está ni en acuerdo ni en desacuerdo.

- Si existiera un medio de información que emitiera datos en tiempo real me haría sentir más seguro.

El $77 \%$ por ciento de la población contestó de manera afirmativa, por lo cual es necesario dar seguimiento a este flujo continuo de información. 


\subsection{Percepción de instituciones}

El $75 \%$ de los encuestados considera que la información no atiende sus necesidades de información en tiempo y forma.

Figura 6: ¿Las instituciones estatales proporcionan información oportuna sobre incidentes delictivos?

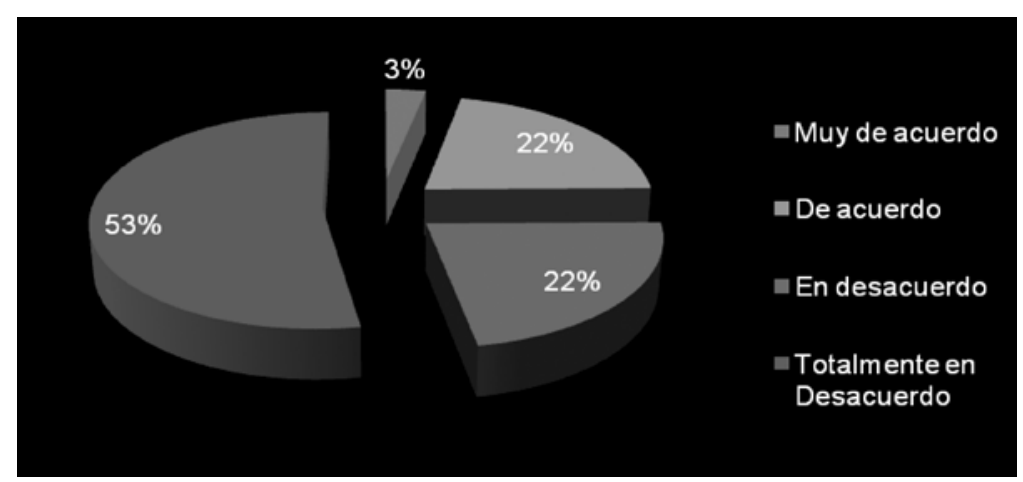

Fuente: Elaboración propia.

En este cuestionamiento se analizó la confianza de la información de hechos delictivos que emite el Ejército, las instituciones estatales y federales. El 49\% de los encuestados confía en la información que emite el ejército; por el contrario un $51 \%$ de los encuestados desconfía de la información que emiten las instituciones estatales y de las instituciones federales un $40 \%$.

Figura 7: Calificación del 1 (máximo) al 5 (mínimo) de la información emitida por las instituciones que participan en la labor de seguridad pública.

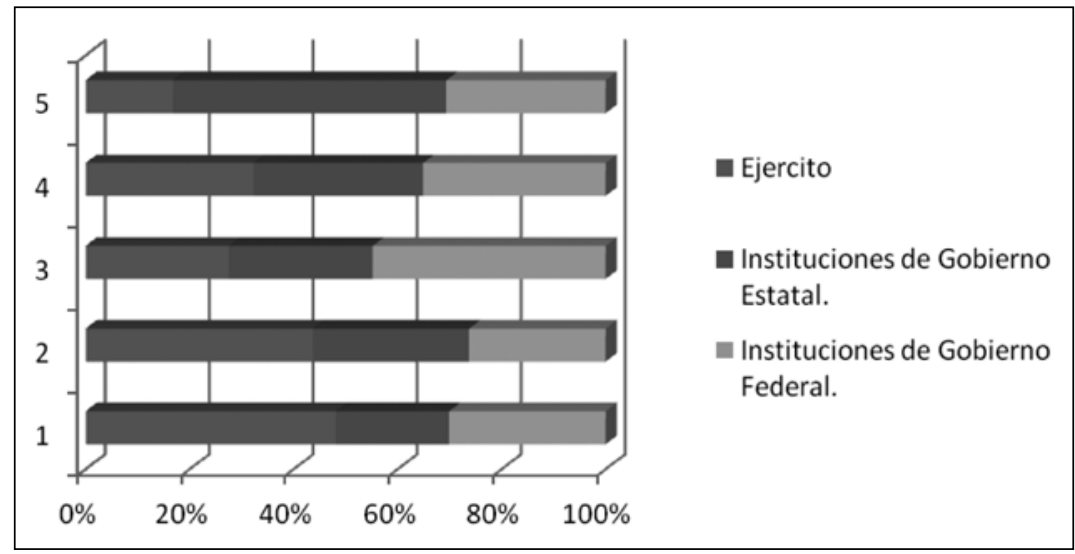

Fuente: Elaboración propia. 


\section{Conclusiones}

En el presente estudio se constata que en el Estado de Tamaulipas (México) se abandonaron los canales y las fuentes formales de comunicación por parte de las instituciones y medios de comunicación. El ciudadano (ante una situación de incertidumbre y sin fuentes formales de comunicación institucional) comienza a alterar sus hábitos, cuestiona los roles y la utilidad de sus organismos de comunicación formales.

A falta de comunicación formal de datos, relacionados con hechos delictivos, los ciudadanos recurren a fuentes informales y redes sociales, las cuales se vuelven fuentes de información $\mathrm{y}$ ofrecen una alta cantidad de testimonios (por parte de los usuarios de las redes sociales) y de desinformación, ya que al menos que existan evidencias tangibles (videos, fotos, etc.) o testigos fidedignos de los hechos (a través de nuestras redes sociales, sean tradicionales o en línea), los hechos no pueden ser corroborados, pero sí reproducidos, causando un impacto en los proceso de percepción y en las actitudes de los actores de este proceso de comunicación. Así, el uso de las nuevas tecnologías va de la mano de las redes sociales tradicionales.

En el caso de la percepción de las instituciones (con relación a la información que brindan) ni las instituciones estatales ni las federales cumplen con la conformidad de la relación brindada, pues los ciudadanos requieren la información al momento del suceso. La institución más “confiable”, según la población encuestada, es el Ejército mexicano.

En cuanto al fenómeno de victimización, debido a la falta de información oficial, es mayor el porcentaje de victimización indirecta que directa. Sin embargo, dentro del modelo de victimización por percepción de SDR, encontramos que el $80 \%$ ha dejado de realizar sus actividades por temor a ser víctima de un delito y el $82 \%$ teme ser víctima de un delito en los próximos seis meses.

\section{Fuentes consultadas}

Amidon, M. (2011). Social Networking: MySpace, Facebook, \& Twitter. Minneapolis: ABDO Publishing Company.

Asociación Mexicana de Internet (2011). "Hábitos de los usuarios de Internet en México". México D. F.: AMPICI, Televisa Interactive Media.

Bernal, C. (2006). Metodología de la investigación para administración, economía, humanidades y ciencias sociales. México D. F.: Pearson Educación.

Botero, E. (2004). Teorías de la comunicación. Santiago de Chile: Universidad de Chile.

Botero, L. y Correa, L. (2006). Teoría de públicos. Lo público y lo privado en la perspectiva de la comunicación. Medellín: Editorial Sello. 
Cancelo Sanmartín. M. y Gadea Aldave, G. (2013). "Empoderamiento de las redes sociales en las crisis institucionales". Vivat Academia, núm. 124, pp. 21-33.

Carvajal, J.; Rodríguez, G. y De Matero, R. (2009). Comunicación informativa y nuevas tecnologías. Buenos Aires: Gran Aldea Editores.

Dewey, J. (2004). La opinión pública y sus problemas. Madrid: Ediciones Morata.

Ellison, B. (2007). "Social Network Sites: Definition, History, and Scholarship". Journal of Computer-Mediated Communication. Vol. 13, núm. 1, pp. 210-230.

Fernández Collado, C. (2001). La comunicación humana en el mundo contemporáneo. México D. F.: Editorial McGraw-Hill.

Ferreiro, X. (2005). La víctima en el proceso penal. Madrid: Ediciones La Ley Actualidad.

Gadea Aldave G. (2014). “Análisis de la imagen institucional de la Secretaría de Relaciones Exteriores de México y su comunicación externa con el segmento poblacional migrante". CienciaUAT. Vol. 21, núm. 3, pp. 65-70.

Goldstein, B. (2006). Sensación y Percepción. Madrid: Ediciones Paraninfo.

González García, M. (1997). La comunicación efectiva. Como lograr una adecuada comunicación en los campos empresarial, social y familiar. México, D. F.: ISEF.

González Herrero, A. (2008). "La gestión proactiva de la comunicación de crisis en la empresa”. En Francescutti, P. (ed.). Comunicación de riesgo, comunicación de crisis. Madrid: Dykinson, Universidad Rey Juan Carlos.

Habermas, J (2009). El futuro de la naturaleza humana. Barcelona: Paidós.

Hart, T.; Greenfield, J. y Haji, D. (2007). People to People Fundraising: Social Networking and Web 2.0 for Charities. Nueva Jersey: Wiley.

Ibáñez, T. (2004). Introducción a la psicología social. Barcelona: Editorial UOC.

La Porte, J.

_(2012). Introducción a la comunicación institucional de la iglesia. Madrid: Colección Pelicano.

_(2005). “Introducción a la comunicación institucional”. Perspectives on Communication, núm. 4, pp. 1-5.

Lazarsfeld, F. (2009). Personal Influence: The Part Played by People in the Flow of Mass Communications. Nueva York: The Free Press. 
Lippman, W. (2010). Public Opinion. Nueva York: Harcourt Brace Jovanovich.

Losada, J. (2010). Comunicación en la gestión de crisis. Lecciones prácticas. Barcelona: Editorial UOC.

Noelle-Neumann, E. (1995). La espiral del silencio. Barcelona: Paidós

Orihuela, J. (2008). “La hora de las redes sociales”. Nueva Revista, núm. 119, pp. 57-62.

Paoli, A. (1987). Comunicación e información. México D. F.: Editorial Trillas.

Rivas, J. (1995). “Desinformación: Revisión de su significado. Del engaño a la falta de rigor”. Estudios sobre el mensaje periodístico, núm. 2, pp. 75-84.

Rodríguez, L. (2003). Victimología. México D. F.: Editorial Porrúa.

Tajfel, H. (1969). "Social and cultural factors in perception”, pp. 315-394. En Lindzey, G. y Aronson, E. (eds.). Handbook of Social Psychology [vol. 3]. Massachusetts: AddisonWesley.

Weber, M. (1984). Los tipos de dominación. México D. F.: Centro Latinoamericano de Economía Humana. 